\title{
Risk of fetal death and malformation related to seizure medications
}

C. Akos Szabo, MD

There is increasing interest regarding the efficacy and safety of seizure medications in special populations, such as in children, the elderly, and pregnant women. The information regarding the safety of newer medications to the embryo and fetus in pregnant women with epilepsy is still sparse, but there is a renewed effort to evaluate the risks in order to better advise women of childbearing age. Several pregnancy registries in the United States, Europe, and Australia are collecting data on the risk of major malformations caused by seizure medications. More information about epilepsy can be found on the next page.

In this issue of Neurology, a study by Meador et al.," titled "In Utero Antiepileptic Drug Exposure: Fetal Death and Malformations," compares the risk of fetal death and congenital malformations for four commonly used seizure medications, including lamotrigine, carbamazepine, phenytoin, and valproic acid. These data are part of an ongoing study looking at short- and long-term developmental effects of seizure medications in children of mothers with epilepsy.

\section{What are the risks of epileptic seizures and seizure medications during pregnancy?}

Fetal exposure to seizure medications may lead to fetal death or congenital malformations, affecting the brain and spinal cord, orofacial cavities, heart, kidneys and urinary tracts, genitals, and skeleton. They are reported to occur in about $6 \%$ of pregnancies in women with epilepsy, constituting twice the risk compared to the general population. To what extent these abnormalities are related to seizures, the underlying epilepsy or psychosocial factors, or seizure medications is unclear. Nonetheless, most women with epilepsy need to be treated during pregnancy. Epileptic seizures are potentially dangerous to the pregnant woman and the fetus. Isolated seizures can lead to falls or other forms of physical injury, while frequent or prolonged seizures can lead to physical stress endangering the health of the woman and fetus.

\section{Are seizure medications necessary during pregnancy?}

As treatment is necessary, which are the medications that are both effective and safe? Most studies have reported on the risk associated with the older seizure medications. These studies included women with uncontrollable seizures who were treated with a combination of seizure medications. In these studies, it was difficult to test the effect of uncontrolled seizures and seizure medications, and which medications posed the greatest risk. Prospective studies are needed, collecting data on women whose seizures are controlled on monotherapy, in order to understand the risks associated with each seizure medication.

\section{What does this study show?}

The study of Meador et al. looked at the outcomes of 333 pregnancies in women who, in large part, were well-controlled on only one seizure medication. This study compared the effects of carbamazepine, phenytoin, lamotrigine, and valproic acid. Women were included if they were otherwise healthy, with a normal intelligence, without a previous history of infection with syphilis or HIV, or recent alcohol or drug abuse. The rate of all serious adverse events was $30(9 \%)$ of 333 pregnancies, with congenital malformations occurring in $22(7 \%)$. The rate of fetal death and congenital malformations was significantly greater with valproic acid $(20 \%)$ when compared to the other agents, including lamotrigine $(1 \%)$, carbamazepine $(8 \%)$, and phenytoin $(11 \%)$. Although lamotrigine had the fewest complications, the difference was not statistically significant compared to carbamazepine and phenytoin. The main limitations of the comparison between the medications less likely to cause fetal malformations are the small number of subjects in each group. Nonetheless, the rate of malformations attributed to valproic acid appears to be dependent on the dose of medication used. If low doses of valproic acid (500 to $1,000 \mathrm{mg}$ / day) can be used to adequately treat seizures, the risk is comparable to other antiepileptic medications.

\section{What does this mean for women with epilepsy who want to become pregnant?}

In summary, there is mounting evidence that the risk for fetal death and congenital malformations in mothers with epilepsy may be affected by the medication they are prescribed. Valproic acid appears to be associated with significantly increased risk for fetal death and congenital malformations, though the risk appears to be dependent on the dose used. Physicians and women of childbearing age both need to be aware of these risks when choosing the appropriate seizure medication during pregnancy. Because women often do not know they are pregnant during the first 4 to 8 weeks of the pregnancy, it is very important to plan the timing of pregnancy very carefully. Women with epilepsy need to have their seizure medications optimized prior to conception, using a single agent which is most effective for their seizure type and at the lowest dose possible. Finally, all women of childbearing age need to be on folate, which has been shown to reduce the risk of malformations in women taking seizure medications.

\section{Reference}

1. Meador KJ, Baker GA, Finnell RH, et al. In utero antiepileptic drug exposure: fetal death and malformations. Neurology 2006;67:407412 . 


\begin{abstract}
What is a seizure?
A seizure is a disruption in the normal electrical activity of the brain. Normally the brain is very active, passing electrical messages back and forth between nerve cells. When a person has a seizure, there is abnormal firing of nerve cells and the messages become jumbled in part or all of the brain.
\end{abstract}

A seizure may cause a variety of different symptoms, such as twitching or shaking in an arm, leg, one side of the face, or the whole body; repetitive movements or gestures; confusion; feelings of fear or other emotions; hallucinations (odd smells, tastes, sounds, or seeing things that are not there); loss of consciousness; and convulsions.

\section{What causes seizures?}

Anyone may have a seizure in certain conditions. Common causes are fever (in young children) (see febrile seizures, below); head trauma (during birth or any time later); infection of the brain or nervous system (e.g., meningitis); brain tumors; very low blood sugar; stroke (a brain attack); lack of oxygen to the brain; and poisoning (e.g., alcohol and various drugs).

\section{What are febrile seizures?}

Febrile seizures are generally benign and occur in children from ages 3 months to 5 years, with average age at onset of 18 to 22 months. They occur in the setting of a febrile illness, usually as the fever is rising fast. They are associated with common childhood infections such as ear infections, tonsillitis, upper airway infections, and gastrointestinal infections. Most are associated with viral illnesses.

Febrile seizures are more common in boys. They also tend to run in families. About one third of children who have a first febrile seizure will have a second one, almost always within 2 years. The earlier the onset of febrile seizures, the greater chance that they will recur. However, fewer than $5 \%$ of children with febrile seizures go on to develop epilepsy.

Although febrile seizures are usually benign, it is critical that the child be evaluated immediately to identify the cause of the fever and treat conditions such as meningitis or intoxication.

\section{What is epilepsy?}

Epilepsy is an episodic recurrence of seizures that are not due to fever, active infection, drug effects, or other triggering causes. It may be caused by a variety of conditions that injure a part or all of the brain, such as problems in development of the brain that occurs before birth, inherited disorders of the brain or nervous system, brain trauma, brain tumors, stroke, infections, and poisoning. About $70 \%$ of cases have no known cause.

Each year there are 125,000 new cases of epilepsy. About 2.5 million people in the United States have some form of epilepsy.

\section{How is epilepsy diagnosed?}

The doctor will need to know as much as possible about what happened during, immediately before, and after the seizure. How often seizures occur, whether there are any warning signs, and whether the patient remembers anything about the seizure are all important. Someone who has witnessed the seizures can provide valuable information that the patient may not know.
Electroencephalography (EEG) is a simple and painless study that records the brain's electrical activity picked up by tiny wires taped to the head. Specific brain wave patterns may be noted during or between seizures in patients with epilepsy and may help with diagnosis.

Imaging studies to look at the brain may be helpful in locating tumors, scars, or other abnormalities that may be causing seizures. Magnetic resonance imaging (MRI) and computed tomography $(\mathrm{CT})$ scans create pictures of the inside of the brain.

\section{How is epilepsy treated?}

Drugs called anticonvulsants are used most commonly to treat seizures. There are a number of different medications that can be used, alone or in combination. These drugs are effective in 60 to $80 \%$ of cases. Close supervision by a doctor is essential to watch for side effects and obtain the best seizure control.

People with epilepsy can help control seizures by avoiding alcohol and caffeine, avoiding unusual stress, getting enough sleep, taking their medications as prescribed, and working closely with their doctor. A special diet may be helpful in controlling certain types of seizures in children but requires very close medical supervision. Surgery may be very helpful when medication fails and the area of the brain where the seizure occurs is known.

\section{For more information}

AAN Foundation:

www.thebrainmatters.com Epilepsy Foundation of America: www.efa.org 


\title{
Neurology
}

\section{Risk of fetal death and malformation related to seizure medications}

\author{
C. Akos Szabo
}

Neurology 2006;67;E6-E7

DOI 10.1212/01.wnl.0000233835.05212.5d

\section{This information is current as of August 7, 2006}

\section{Updated Information \&} Services

References

Citations

Permissions \& Licensing

Reprints including high resolution figures, can be found at: http://n.neurology.org/content/67/3/E6.full

This article cites 1 articles, 1 of which you can access for free at: http://n.neurology.org/content/67/3/E6.full\#ref-list-1

This article has been cited by 1 HighWire-hosted articles: http://n.neurology.org/content/67/3/E6.full\#\#otherarticles

Information about reproducing this article in parts (figures,tables) or in its entirety can be found online at:

http://www.neurology.org/about/about_the_journal\#permissions

Information about ordering reprints can be found online:

http://n.neurology.org/subscribers/advertise

Neurology ${ }^{\circledR}$ is the official journal of the American Academy of Neurology. Published continuously since 1951, it is now a weekly with 48 issues per year. Copyright . All rights reserved. Print ISSN: 0028-3878. Online ISSN: 1526-632X.

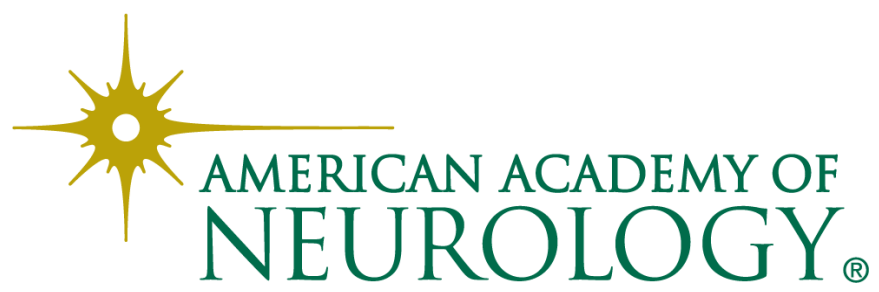

